Japanese Pychological Research

1986 , Vol. 3 , No. 3, $139-148$

\title{
Similarity of allocation and interpersonal attraction under cooperative and competitive conditions ${ }^{1}$
}

\author{
HIDETAKI OKLDA \\ Department of Social Psvchology, Faculty of Letters, University of Tokyo:
}

\begin{abstract}
The present study explored the relations between opinion similarity and interpersonal at traction among persons who advocated either equal or equitable allocation of rewards in cooperative or competitive situations. The main hypotheses were that subjects would prefer equality to equity in cooperative situations while they would prefer equity to equality in competitive situations, and that subjects would like members who favored similar allocation better than those who favored dissimilar allocation. One hundred and twenty undergraduate students participated in a dilemma game in groups of four under either cooperative or competitive conditions in Experiment 1. Each subject was informed of the opinion on reward allocation of one of his group members. The main hypotheses were supported. Further, altruistic members were better liked than self-interested members, whether or not their opinions were similar to those of the subjects. Similar results were obtained in Experiment 2 in which 168 undergraduate subjects observed the game.
\end{abstract}

Key words: attitude similarity, competition, cooperation, distributive justice, equality, equity, interpersonal attraction.

It has been assumed that fair allocators are better liked than unfair allocators (Ajzen, 1982). However, as fair allocation is in the eye of the beholder, whether an allocator is liked or not would depend not only upon the allocator but also upon the person who judges him. The present study was based on the assumption that similarity of opinion would affect interpersonal attraction toward others in the allocation of rewards. As Byrne (1971) has demonstrated, subjects prefer others who hold similar opinions to those who hold dissimilar opinions. Correspondingly, when allocating rewards among group members, subjects would prefer other members favoring similar allocation to those favoring dissimilar allocation.

In Western society, equity and equality are primary fairness rules (Keil \& McClin-

1 This study was supported by a Grant-in-Aid for Scientific Researches, Ministry of Education, Science and Culture, No. 58710036.

2 Correspondence concerning this article should be addressed to Hidetaka Okuda, who is now at the Department of Sociology, Chukyo University, 101 Tokodate, Kaizu-cho, Toyota, Aichi 470-03. tock, 1983). Apparently, equity works to the advantage of high-input members and equality works to the advantage of lowinput members, even though the rewards of the group as a whole remain constant. Kahn, Lamm, and Nelson (1977) found that generous persons such as high-input equal and low-input equitable allocators were well liked but stingy persons such as high-input equitable and low-input equal allocators were not so well liked. Similar results were obtained not only in the U.S. (Brickman \& Bryan, 1976; Greenberg, 1982; Kahn et al., 1977; Watts \& Messé, 1982) but also in Australia (Feather \& O'Driscoll, 1980), Hong Kong (Leung \& Bond, 1984), Japan (Okuda, 1984), and West Germany (Kahn et al., 1977). The important point here is that Kahn et al. (1977) reported equal allocators were not necessarily liked more than equitable allocators though most subjects favored equality rather than equity. This findings implies that subjects' opinions on allocation had little effects on their attraction for allocators. However, Okuda (1984, 1985 ) found that similarity of allocation 
choices had something to do with subjects attraction for others.

The opinions on allocation would be allected by situational variables. Deutsch (1975) assumed that people tend to use equality rather than equity in cooperative situations, and tend to use equity rather than equality in competitive situations. Schwinger (1980) found some support for this belief, but the results were rather equivocal because both high- and low-input subjects showed the tendency to choose altruistic allocation which is common for allocators (Mikula, 1974).

The question is whether subjects' opinions on allocation under cooperative or competitive circumstances would affect their liking for members who advocated their opinions on allocation. Kiesler (1966) found that the partners whose inputs were higher than subjects were liked when they allocated rewards cqually rather than equitably under cooperative circumstances, while the opposite trend appeared under competitive circumstances. However, as the partners' inputs were always higher than those of subjects, they tended to be liked under both cooperative and competitjve circumstances when they allocated equally rather than equitably. Therefore, it is not clear in Kiesler's experiment whether subjects liked equal partners because allocation was in accord with role requirement or because the allocation was to the advantage of subjects. The present study employed medium-input subjects and observer subjects so as to avoid such ambiguity. Thus, they would favor equality under cooperation and equity under competition. As a result, they would like members who chose similar allocation and dislike members who chose dissimilar allocation.

To test the above implications, the present study examined the following hypotheses. In a group whose members cooperate with one another, subjects would prefer equality to equity. Therefore, sub- jects would tend to like members who favored equality and tend to dislike those who favored equity. In a group whose members compete with one another, subject would prefer equity to equality. Therefore, subjects would like members who favored equity and dislike those who favored equality.

\section{Experiment 1}

\section{Method}

Overview. Groups of four subjects participated in a procedure ostensibly designed to explore decision-making in a dilemma game. Half of the subjects took part in a cooperative game, while the remaining half of the subjects took part in a competitive game. After the game was over, each subject received false scores for the four members of his group. Regardless of his actual performance during the game, each subject was informed that he and another member of his group had achieved average scores, while one other member had achieved a higher score and the remaining member a lower score. Then the subjects were asked to express their opinions on the allocation of the reward in writing, and to pass around the papers upon which the opinions were written amongst themselves. Next, each subject was provided with one of the others' opinions. His opinion favored either equality or equity, and his score was either high, medium or low. Finally, the subjects were asked their impression of him anonymously. Thus, the design was a 2 (game situation) $\times 3$ (inputs of target member) $\times$ 2 (allocation of target member) factorial design with 10 subjects in each of the 12 cells.

Subjects. One hundred and twenty male undergraduate students who were enrolled in an introductory psychology course at the University of Tokyo volunteered as subjects. Each subject composing groups of four members was randomly assigned to each game situation. 
Procedure. Subjects participalcal in the experiment in groups of four. They were strangers to one another. At lirst, eatch subject was placed in one ol lour separate booths so arranged that subjects could not communicate with each other cither visual ly or verbally. Subjects were told that each booth was assigned a letter from $\mathrm{A}$ to $\mathrm{D}$ at random, so that none of them could identify who had which letter. Subjects were also asked not to speak until the end of the experiment.

The task was a four-person dilemma game. During the game period each subject was asked to choose either "red" or "white". The choice was made 10 times by each subject on a signal from the experimenter. Whenever a subject chose white, he received one point. When a subject chose red, however, the points given to him depended upon the other subjects' choices. A subject received two points if he was the only person choosing red among the four members in his group. He received one point if he and one other member chose red. He received zero points if three or all four members including him chose red. Each subject was allowed to chose red for fewer than five trials. Therefore, the maximum points he could get were 15 and minimum were 5 . In fact, each subject received false feedback after the game, and found that he had received 10 .

Finally, the subjects were asked to respond to the questionnaires. Then they were interviewed and fully debriefed. They were also asked not to mention any part of the experiment to the other students.

Independent variables. The independent variables in this study were the game situation, the target member's inputs, and the target member's opinion on allocation. These variables formed a $2 \times 3 \times 2$ factorial design.

[Game situation] Manipulation of the game situation was accomplished at the beginning of the game according to the in- structions of the experimenter. Under cooperative conditions, subjects were encouraged to get as many group points as possible. The experimenter told them as lollows:

The purpose of the present game is to maximize group scores. The goal is to get a higher group score than any other group, so please try to get as many points as possible.

By contrast, subjects were encouraged to get as many individual points as possible under competitive conditions. The experimenter told them as follows:

The purpose of the present game is to maximize individual scores. The goal is to get a higher individual score than any other member, so please try to get as many points as possible.

The above procedure was employed to produce intergroup competition under cooperative conditions and intragroup competition under competitive conditions.

[Inputs of target member] Soon after the game, each subject received all four members' scores. Each subject learned that he and another member had 10 points, a third member had 12, and the fourth member had 8 . Under high-input conditions, the target member was the one who got 12 points. Under medium- and lowinput conditions, the target member got 10 and 8 points respectively.

[Allocation of target member] When the game was over and scores of the game were given to the subjects, the experimenter asked them to write their opinions on allocation down on paper and to exchange these amongst themselves. In order to elicit subjects' general opinions on allocation, neither the amount nor the nature of the reward was clearly mentioned. The questionnaire contained the following allocation alternatives: (a) winner-take-all, (b) loser-take-all, (c) equal allocation, (d) equitable allocation, (e) compromise between equal and equitable allocation, and (f) free responses. Subjects were asked to choose the one which most nearly corre- 
sponded to their opinions on reward allocation.

Having collected the questionnaires, the experimenter distributed a prepared opinion which was disguised as one of the members' opinions on allocation. Under equality conditions, the target member had chosen equal allocation which stated that the reward should be divided evenly among all members. Under equity conditions, the target member had chosen equitable allocation which stated that the reward should be divided in proportion to each member's score.

Dependent measures. At the end of the experiment, subjects were asked to respond to the questionnaire which was designed to assess each subject's evaluation of the target member. Subjects responded to Interpersonal Judgement Scale (Byrne, 1971), and the last two items (personal feelings and working together in an experiment) were summed to produce a overall measure of interpersonal attraction. Subjects were also asked to respond to other questions for manipulation checks and fairness of the target member's opinion.

\section{Results}

Manipulation checks. The effectiveness of game situation manipulation was assessed with a multiple choice question which asked subjects to choose one of three alternatives. These were that the game situation was most likely to be: (a) cooperative, (b) competitive, or (c) individualistic. Under cooperative conditions, subjects who decided that the game was cooperative, competitive, or individualistic were 36,3 , and 21 , respectively. On the other hand, subjects who chose these alternatives under competitive conditions were 9 , 30 , and 21 , respectively. A chi-square test revealed that the difference was significant, $\chi^{2}=27.3, d f=2, p<.001$.

The manipulation of the inputs of the target member was also successful. Subjects were asked the degree to which the
Table 1

Allocation choices of subjects as a function of target member's allocation and game situation in Lxp. 1

\begin{tabular}{cccc}
\hline & \multicolumn{3}{c}{ Allocation by subjects } \\
\cline { 2 - 4 } Situation & Equality & Compromise & Equity \\
\hline Cooperation & & & \\
Equality & 22 & 6 & 2 \\
Equity & 23 & 6 & 1 \\
Competition & & & \\
Equality & 11 & 7 & 12 \\
Equity & 13 & 8 & 9
\end{tabular}

Note: Cell entries indicate the number of subjects.

Table 2

Evaluation for target member as a function of his inputs, his allocation, and game situation in Exp. 1

\begin{tabular}{cccc}
\hline & \multicolumn{3}{c}{ Inputs } \\
\cline { 2 - 4 } Situation & High & Medium & Low \\
\hline & Interpersonal Attraction & \\
\hline $\begin{array}{c}\text { Cooperation } \\
\text { Equality }\end{array}$ & $9.4(10)$ & $9.6(10)$ & $9.1(10)$ \\
Equity & $6.7(10)$ & $8.6(10)$ & $9.1(10)$ \\
Competition & & & \\
Equality & $9.2(10)$ & $8.8(10)$ & $8.4(10)$ \\
Equity & $7.9(10)$ & $8.1(10)$ & $9.7(10)$ \\
\hline
\end{tabular}

Fairness of Allocation b)

$\begin{array}{cccc}\text { Cooperation } & & & \\ \text { Equality } & 4.9(10) & 6.2(10) & 5.2(10) \\ \text { Equity } & 3.6(10) & 4.3(10) & 4.8(10) \\ \text { Competition } & & & \\ \text { Equality } & 4.6(10) & 4.4(10) & 4.8(10) \\ \text { Equity } & 4.6(10) & 4.8(10) & 4.9(10)\end{array}$

Note: Number of subjects is shown in parentheses. a) Higher cell entries indicate higher interpersonal attraction on a scale ranging from 2 to 14 .

b) Higher cell entries indicate fairer allocation on a scale ranging from 1 to 7 .

target member's score was high or low on a 7-point scale. Means of high-, medium-, and low-input conditions are 5.44, 3.95, and 2.73 , respectively. The analysis of variance revealed that the difference was significant, $F(2,108)=276.1, p<.0001$. 
The manipulation of the allocation of the target member was also successful. No one responded incorrectly to the question which asked subjects to choose the target member's opinion among the allocation alternatives.

Subjects' allocation choices. Subjects expressed their opinions on allocation publicly in the first questionnaire by choosing one among the given allocation alternatives. A chi-square test revealed that the difference between cooperative and competitive conditions was significant, $\chi^{2}=$ 10.1, $d f=2, p<.01$. These results were presented in Table 1. Among the 60 subjects participating under each condition, those who chose equal allocation were 45 and 24 respectively, while those who chose equitable or compromise allocation were 15 and 36 respectively.

Interpersonal attraction. Attraction for target member was examined by means of analysis of variance using Situation, Inputs, and Allocation as independent variables. The analysis revealed that the main effect of Allocation, $F(1,108)=5.96, p<$ .05 , and the interaction effect of Allocation $\times$ Inputs, $F(2,108)=6.52, p<.01$, were significant. The predicted interaction effect of Situation $\times$ Allocation was not significant. These results are presented in Table 2. High-input members were liked better when they favored equal rather than equitable allocation, while low-input members were liked better when they favored equitable rather than equal allocation. On the other hand, medium-input members were liked better when they favored equal rather than equitable allocation under both cooperative and competitive conditions (n.s. by Duncan Multiple Range Test).

In order to elaborate the relations between similarity of subjects' and target members' opinions and interpersonal attraction, a four factorial analysis of variance was performed adding Subjects' Allocation Choices as an independent variable. Subjects who chose compromise al-
Table 3

Waluation for target member as a function of his allocation, game situation, and subjects' allocation choices in Exp. 1

\begin{tabular}{|c|c|c|}
\hline \multirow[b]{2}{*}{ Situation } & \multicolumn{2}{|c|}{ Allocation by subject } \\
\hline & Equality & Lquity \\
\hline \multicolumn{3}{|c|}{ Interpersonal Attraction ${ }^{\text {a }}$} \\
\hline \multicolumn{3}{|l|}{ Cooperation } \\
\hline Equality & $9.4(22)$ & $9.3(8)$ \\
\hline Equity & $8.1(23)$ & $8.3(7)$ \\
\hline \multicolumn{3}{|l|}{ Competition } \\
\hline Equality & $9.6(11)$ & $8.4(19)$ \\
\hline Equity & $7.6(13)$ & $9.3(17)$ \\
\hline \multicolumn{3}{|c|}{ Fairness of Allocation b } \\
\hline \multicolumn{3}{|c|}{ Cooperation } \\
\hline Equality & $5.9(22)$ & $4.1 \quad(8)$ \\
\hline Lquity & $3.9(23)$ & $5.4(7)$ \\
\hline \multicolumn{3}{|l|}{ Competition } \\
\hline Equality & $5.4(11)$ & $4.2(19)$ \\
\hline Equity & $4.0(13)$ & $5.4(17)$ \\
\hline
\end{tabular}

Note: Number of subjects is shown in parentheses. a), br See Table 2.

location were pooled in equity, because they had similar tendencies. The analysis revealed that the main effect of Allocation was significant, $F(1,96)=3.94, p<.05$. There were also significant interaction effects of Allocation $\times$ Inputs, $F(2,96)=6.86$, $p<.01$, Allocation $\times$ Subjects' Allocation, $F(1,96)=10.86, p<.05$, and Situation $\times$ Allocation $\times$ Subjects' Allocation, $F(1,96)$ $=4.26, p<.05$. These results were presented in Table 3 . Not only did subjects like altruistic members better than selfinterested members, but they evaluated the members of similar opinions more positively than those of dissimilar opinions under competitive conditions while they liked equal rather than equitable members better under cooperative conditions.

Fairness of allocation. Subjects were questioned about the degree to which the opinion of the target member was fair. The results were examined by means of analysis of variance using Situation, Input, and Allocation as independent variables. The 
analysis reveted that the main effect of Nllocalion, $F(1,108)=5.79, p<.05$, and the interaction eflect of Allocation $\times$ Situation, $F(1,108)=10.12, p<.01$, were significant. These results are presented in 'Table 2. Liqual opinion was rated as fairer under cooperative conditions than under competitive conditions, while equitable opinion was rated as fairer under competitive conditions than under cooperative conditions.

In order to elaborate the relations between similarity of opinions and fairness, a four factorial analysis of variance was performed adding Subjects' Allocation Choices as an independent variable. The analysis indicated the significant main effect of Inputs, $F(2,96)=6.08, p<.01$, and interaction effect of Allocation $\times$ Subjects' Allocation, $F(1, \quad 96)=77.97, \quad p<.001$. These results were presented in Table 3. Subjects evaluated similar opinions to be fairer than dissimilar opinions.

\section{Experiment 2}

Subjects who participated in Experiment 1 made their opinions public for both other subjects and experimenters. Such public opinions might have been affected by expectations from others (Reis \& Gruzen, 1976). One way to avoid such a possibility is for subjects not to make their opinions public but to make them private. For that purpose, subjects of Experiment 2 did not participated but observed the game, and answered their opinions privately. The predictions were the same as the first experiment, although subjects in the former experiment were participants in the actual game while subjects in the latter one were observers of the game.

\section{Method}

Subjects. One hundred and sixty-eight undergraduate students who were enrolled in an introductory psychology course at Sensyu University served as subjects. Most of them were male freshmen.
All of the subjects assembled in a large classroom, at which time an questionnaire booklet was passed out to each of the subjects. The duration of the experiment from start to finish was about thirty minutes.

Procedure. Each subject was presented with a description of one of the game conditions in Experiment 1. In short, the game was a kind of four-person dilemma game. Half of the subjects were presented with a cooperative game situation, while the remaining half of the subjects were presented with a competitive game situation. After the game was over and the scores of the four members were made public, one member expressed an opinion on allocation. His opinion favored either equality or equity, and his score was either high, medium, or low. After reading the description, subjects were asked to respond to some questions.

Independent and dependent variables. The independent variables were the same as those of the Experiment 1. These formed a 2 (cooperative or competitive game situation) $\times 3$ (inputs of target member) $\times 2$ (allocation of target member) factorial design with 14 subjects in each of the 12 cells.

Dependent variables were also similar to those of Experiment 1. However, compromise allocation was not included among the allocation alternatives. The allocation alternatives were: (a) winner-take-all, (b) loser-take-all, (c) equal allocation, (d) equitable allocation, and (e) free responses. Fairness of the target member's opinion was not included in the questionnaire.

\section{Results}

Manipulation checks. The manipulation of the game situation was successful. Subjects under cooperative conditions who decided that the game situation was cooperative, competitive, or individualistic were 33,8 , and 43 , respectively, while those who chose these alternatives under competitive conditions were 3,27 , and 54 , 
respectively. A chi-square test revealed that the difference was significant, $\chi^{2}$ $36.56, d f=2, p<.001$.

The manipulation of the target member's inputs was also successful. Subjects were asked the degree to which target member's score was high or low on a 5point scale. Means of the high-, medium-, and low-input conditions were $3.8,3.3$, and 2.6, respectively. Analysis of variance indicated that the difference was significant, $F(2,156)=45.6, p<.001$.

The manipulation of the allocation of the target nember was also successful. No one responded incorrectly to the question which asked subjects to choose the target members' allocation among the allocation alternatives.

Subjects' allocation choices. Subjects responded to the question which asked their own opinions on allocation by choosing one of the allocation alternatives. A chisquare test revealed that the difference between cooperative and competitive conditions was significant, $\chi^{2}=38.88, d f=2$, $p<.001$. These results are presented in Table 4. Subjects who chose equal allocation under each condition were 68 and 29 respectively, while those who chose equitable allocation were 13 and 37 respectively. Most of the subjects who chose something other than equality or equity chose winner-take-all allocation.

Interpersonal attraction. The analysis of variance of attraction for target member revealed that the main effect of Allocation, $F(1,156)=7.13, p<.01$, and the interaction effect of Allocation $\times$ Inputs, $F(1,156)=15.1, p<.001$, were significant. These results are presented in Table 5 . High-input members were liked when they favored equal rather than equitable allocation, while low-input members were liked when they favored equitable rather than equal allocation. Medium-input members were liked, however, when they favored equal rather than equitable allocation under both cooperative and competitive conditions ( 8.9 vs. $7.1, p<.05$ by Duncan
Table 4

Allocation choices of subjects as a function of target member's allocation, and game situation in lixp. 2

\begin{tabular}{lccc}
\hline & \multicolumn{3}{c}{ Allocition by subjects } \\
\cline { 2 - 3 } Situation & Equality & Equity & Others \\
\hline Cooperation & & & \\
Equality & 33 & 7 & 2 \\
Equity & 35 & 6 & 1 \\
Competition & & & \\
Equality & 16 & 17 & 8 \\
Equity & 13 & 20 & 9
\end{tabular}

Note: Cell entries indicate the number of subjects.

Table 5

Interpersonal attraction toward target member as a function of his inputs, his allocation, and game siuation in Fxp. 2

\begin{tabular}{lll}
\hline & \multicolumn{2}{c}{ Situation } \\
\cline { 2 - 3 } Allocation & Cooperation & Competition \\
\hline Equality & & \\
High inputs & $9.9^{\circ}(14)$ & $9.8(14)$ \\
Medium inputs & $8.9(14)$ & $8.8(14)$ \\
Low inputs & $8.1(14)$ & $8.0(14)$ \\
Equity & & \\
High inputs & $6.9(14)$ & $8.1(14)$ \\
Medium inputs & $7.1(14)$ & $8.4(14)$ \\
Low inputs & $9.5(14)$ & $8.9(14)$
\end{tabular}

Note: Number of subjects is shown in parentheses. a) See Table 2.

Multiple Range test).

In order to elaborate the relations between opinion similarity and interpersonal attraction, a three factorial analysis of variance was performed using Subjects' Allocation Choices as an independent variable instead of Situation. A four factorial analysis of variance was abandoned because of zero cell entries. Subjects favoring something other than equality or equity were excluded from this analysis. The analysis revealed that there were significant interaction effects of Allocation $x$ Inputs, $F(2,135)=11.01, p<.001$, and Allocation $\times$ Subjects' Allocation, $F(1,135)$ $=16.31, p<.001$. These results are pre- 
Table 6

Interpersonal attraction toward target member as a function of his inputs, his allocation and subjects' allocation choices in Exp. 2

\begin{tabular}{lcc}
\hline & \multicolumn{2}{c}{ Allocation by subject } \\
\cline { 2 - 3 } Allocation & Equality & Equity \\
\hline Equality & & \\
High inputs & $10.6^{\text {a) }}(19)$ & $9.0(3)$ \\
Medium inputs & $9.5(12)$ & $8.6(13)$ \\
Low inputs & $8.1(18)$ & $7.8(8)$ \\
Equity & & \\
High inputs & $7.3(20)$ & $8.3(7)$ \\
Medium inputs & $7.2(16)$ & $8.7(6)$ \\
Low inputs & $8.7(12)$ & $9.8(13)$
\end{tabular}

Notes: Number of subjects is shown in parentheses. *) Sce Table 2 .

sented in Table 6 . In addition to the fact that subjects liked altruistic members better than self-interested members, they evaluated the members of similar opinion more positively than those of dissimilar opinion.

\section{Discussion}

The apparent belief of the subjects that the game was individualistic must have reflected the fact that it was not the scores but rather the decisions which were made individualistically under both cooperative and competitive conditions. While subjects were formulating their opinions on allocation, they must necessarily have taken into account whatever factors were appropriate as inputs. As the members under cooperative conditions tried not only to increase their own scores but to increase other members' scores, subjects must have perceived that members' inputs were not only their individual scores but also their group scores. On the other hand, as the members under competitive conditions tried to increase their individual scores, subjects must have perceived that members' inputs were only their individual scores. These differences must have affected subjects' opinions on allocation.
Subjects chose equal allocation over equitable allocation under cooperative conditions, but tended to make the opposite choice under competitive conditions in both Experiments $I$ and 2, although subjects in Experiment 1 were participants and made their opinions known to other members while those in Experiment 2 did not.

The present study confirmed past findings, which established that altruistic persons were liked but self-interested persons were not. That is, subjects like equal members of high-inputs and equitable members of low-inputs, but dislike equal members of low-inputs and equitable members of high-inputs (Feather \& O'Discoll, 1980; Greenberg, 1982; Kahn et al., 1977; Leung \& Bond, 1984; Okuda, 1984; Watts \& Messé, 1982). However, the reasons why altruistic or generous allocators are better liked than self-interested or parsimonious allocators have not been made clear. One of the possible reasons is that altruistic allocation is fairer than self-interested allocation. For example, Feather and O'Driscoll (1980) found that high- and low-input modest allocators were not only liked but also judged to be fairer than self-interested allocators. Similar results were reported by Watts and Messé (1982) for high-input members, and Leung and Bond (1984). However, Greenberg (1982) found that high-input members were judged to be fairer when they allocated reward equitably rather than equally, but the latter tended to be liked better than the former. The results of Experiment 1 suggest that though fairness and altruism are both related to interpersonal attraction, fairness was related to allocation similarity but altruism was not. Okuda (1985) also found that allocation similarity was more closely related to fairness judgment than to interpersonal attraction. These imply that fair allocation and altruistic allocation have different functions.

Medium-input members must be nei- 
ther altruistic nor self-interested when they favor equality or equity. Therefore, they were judged to be fairer when they favored equality rather than equity under cooperative conditions, and vice versa under competitive conditions. However, they tended to be liked better when they favored equality rather than equity under both cooperative and competitive conditions. Leventhal (1976) has suggested that equality is used when the allocators are desirous of preventing intragroup conflict and maintaining a high level of harmony and satisfaction among group members, while equity is used when the allocators are primarily interested in maximizing the performance of group members. Kahn et al. (1977) suggests that members who try to maintain group harmony would be seen in a more positive light than those who try to maximize performance. But when opinion similarity was held constant, the tendency for equal members to be liked was diminished, especially in Experiment 2. This implies that the tendency above was limited as long as subjects preferred equality to equity.

The present study revealed that both interpersonal attraction and fairness judgement are related to opinion similarity. Moreover, the latter has a closer relation with opinion similarity than the former. It seems that fairness of the target members' opinions on allocation was determined mainly by opinion similarity, while interpersonal attraction was determined by both similarity and altruism of the members. These interpretations must be tested by future researches. It is hoped that the present study not only presents a framework for the study of interpersonal attraction under cooperation and competition, but also offers a direction for the pursuit of a better understanding of such relations.

\section{References}

Ajzen, I. 1982 Equity in attitude formation and change. In J. Greenberg \& R. L. Gohen (Eds.), Equity and justice in social behavior. New York: Academic Press. Pp. 161-186.

Brickman, P., \& Bryan, J. H. 1976 Equity versus equality as factors in children's moral judgements of thefts, charity, and third-party transfers. Journal of Personality and Social Psychology, 34, 757761.

Byrne, D. 1971 The altraction paradigm. New York: Academic Press.

Deutsch, M. 1975 Equity, equality and need: What determines which value will be used as the basis of distributive justice? Journal of Social Issues, 31, 137-149.

Feather, N. T., \& O'Driscoll, M. P. 1980 Observer's reactions to an equal or equitable allocator in reaction to allocator input, causal attributions, and value importance. European Journal of Social Psychology, 10, 107-129.

Greenberg, J. 1982 Countering inequity with inequity: Over-rewarding generosity and underrewarding greed. European Joumal of Social Psychology, 12, 181-185.

Kahn, A., Lamm, H., \& Nelson, R. E. 1977 Preferences for an equal or equitable allocator. Journal of Personality and Social Psychology, 35, 837-844.

Keil, L. J., \& McClintock, C. G. 1983 A developmental perspective on distributive justice. In D. M. Messick \& K.S. Cook (Eds.), Equtity theory: Psychological and sociological perspectives. New York: Praeger. Pp. 13-46.

Kiesler, S. B, 1966 The effect of perceived role requirements on reactions to favor-doing. Journal of Experimental Social Psychology, 2, 198-210.

Leung, K., \& Bond, M. H. 1984 The impact of cultural collectivism on reward allocation. Journal of Personality and Social Psychology, 47, 793-804.

Leventhal, G.S. 1976 Fairness in social relationships. In J. Thibaut, J. Spence, \& R. Carson (Eds.), Contemporary topics in social psychology. Morristown, N.J.: General Learning Press. Pp. 211-239.

Mikula, G. 1974 Nationality, performance, and sex as determinants of reward allocation. Journal of Personality and Social Psychology, 29, 435-440.

Okuda, H. 1984 Opinion on allocation and its effect on interpersonal attraction. Japanese Journal of Psychology, 55, 22-28. (In Japanese with English abstract)

Okuda, H. 1985 Self-interest in reward allocation and interpersonal attraction. Japanese Journal of Psychology, 56, 153-166. (In Japanese with English abstract)

Reis, H. T., \& Gruzen, J. 1976 On mediating 
equity, equality, and self-interest: The role of self-presentation in social exchange. Journal of Experimental Social Psychology, 12, 487-503.

Schwinger, T. 1980 Just allocations of goods: becision among three principles. In G. Mikula (Ed.), Justice and social interaction. Bern: Hans Huber. Pp. 95-126.
Watts, B. L., \& Messé, L. A. 1982 The impact of task inputs, situational context, and sex on evaluations of reward allocators. Social Psychology Quarterly, 45, 254-262.

(Received July 16, 1985; accepted March 8, 1986) 